\title{
List Supermodular Coloring with Shorter Lists
}

\author{
Yu Yokoi *
}

October 14, 2018

\begin{abstract}
In 1995, Galvin proved that a bipartite graph $G$ admits a list edge coloring if every edge is assigned a color list of length $\Delta(G)$, the maximum degree of the graph. This result was improved by Borodin, Kostochka and Woodall, who proved that $G$ still admits a list edge coloring if every edge $e=s t$ is assigned a list of $\max \left\{d_{G}(s), d_{G}(t)\right\}$ colors. Recently, Iwata and Yokoi provided the list supermodular coloring theorem, that extends Galvin's result to the setting of Schrijver's supermodular coloring. This paper provides a common generalization of these two extensions of Galvin's result.
\end{abstract}

\section{Introduction}

An edge coloring of an undirected graph ${ }^{1}$ is a function that assigns a color to each edge so that no two adjacent edges have the same color. In 1995, Galvin [5] proved the list edge coloring conjecture for bipartite graphs, which states that the list edge chromatic number of a bipartite graph equals its edge chromatic number. Since König's theorem [7] states that the edge chromatic number of a bipartite graph $G$ equals its maximum degree $\Delta(G)$, Galvin's result is written as follows.

Theorem 1.1 (Galvin [5]). For a bipartite graph $G$, if each edge e has a list $L(e)$ of $\Delta(G)$ colors, then there exists an edge coloring such that every edge $e$ is assigned a color in $L(e)$.

Exploiting Galvin's proof technique for Theorem 1.1. Borodin, Kostochka and Woodall [2] showed the following stronger version, in which some elements may have shorter lists. For a bipartite graph $G$ and a vertex $s$, we denote by $d_{G}(s)$ the degree of $s$ in $G$.

Theorem 1.2 (Borodin, Kostochka and Woodall [2]). For a bipartite graph $G=(S, T ; E)$, if each edge $e=$ st has a list $L(e)$ of $\max \left\{d_{G}(s), d_{G}(t)\right\}$ colors, then there exists an edge coloring such that every edge $e$ is assigned a color in $L(e)$.

Another generalization of Galvin's result is the list supermodular coloring theorem of Iwata and Yokoi [6]. It extends Theorem 1.1 to the setting of Schrijver's supermodular coloring [8], which is described below. (We mostly use the same notations as in [6].)

Let $U$ be a finite set. We say that $X, Y \subseteq U$ are intersecting if none of $X \cap Y, X \backslash Y$ and $Y \backslash X$ are empty. A family $\mathcal{F} \subseteq 2^{U}$ is called an intersecting family if every intersecting pair of $X, Y \in \mathcal{F}$ satisfies $X \cup Y, X \cap Y \in \mathcal{F}$. A set function $g$ on $\mathcal{F}$ is called intersecting-supermodular if $\mathcal{F}$ is an intersecting family and $g$ satisfies the supermodular inequality $g(X)+g(Y) \leq g(X \cup Y)+g(X \cap Y)$ for every intersecting pair of $X, Y \in \mathcal{F}$. For any positive integer $k \in \mathbf{N}$, we write $[k]:=$ $\{1,2, \ldots, k\}$. A function $\pi: U \rightarrow[k]$ dominates $g: \mathcal{F} \rightarrow \mathbf{Z}$ if $|\pi(X)| \geq g(X)$ holds for every $X \in \mathcal{F}$, where $\pi(X):=\{\pi(u) \mid u \in X\}$.

\footnotetext{
*National Institute of Informatics, Tokyo 101-8430, Japan. E-mail: yokoi@nii.ac.jp.

${ }^{1}$ In this paper, a bipartite graph means a bipartite multigraph, i.e., parallel edges are allowed.
} 
Let $g_{1}: \mathcal{F}_{1} \rightarrow \mathbf{Z}$ and $g_{2}: \mathcal{F}_{2} \rightarrow \mathbf{Z}$ be intersecting-supermodular functions. A function $\pi: U \rightarrow[k]$ is called a supermodular $k$-coloring for $\left(g_{1}, g_{2}\right)$ if it dominates both $g_{1}$ and $g_{2}$. Let us assume $|X| \geq g_{i}(X)$ for every $i \in\{1,2\}$ and $X \in \mathcal{F}_{i}$. This condition is clearly necessary for the existence of a supermodular $k$-coloring. Schrijver [8] showed that, under this assumption, the minimum $k \in \mathbf{N}$ that admits a supermodular $k$-coloring of $\left(g_{1}, g_{2}\right)$ equals $\Delta\left(g_{1}, g_{2}\right)$, where

$$
\Delta\left(g_{1}, g_{2}\right):=\max \left\{1, \max \left\{g_{i}(X) \mid i \in\{1,2\}, X \in \mathcal{F}_{i}\right\}\right\} .
$$

As a list coloring version of the Schrijver's result, Iwata and Yokoi [6] proved the following list supermodular coloring theorem. Let $\Sigma$ be a set of colors and $L: U \rightarrow 2^{\Sigma}$ be an assignment of color lists to elements. A function $\varphi: U \rightarrow \Sigma$ is called a list supermodular coloring for $\left(g_{1}, g_{2}, L\right)$ if $\varphi$ dominates both $g_{1}$ and $g_{2}$ and every $u \in U$ satisfies $\varphi(u) \in L(u)$.

Theorem 1.3 (Iwata and Yokoi [6]). Let $g_{1}: \mathcal{F}_{1} \rightarrow \mathbf{Z}$ and $g_{2}: \mathcal{F}_{2} \rightarrow \mathbf{Z}$ be intersectingsupermodular functions such that $|X| \geq g_{i}(X)$ for every $i \in\{1,2\}$ and $X \in \mathcal{F}_{i}$. If each element $u \in U$ has a list $L(u)$ of $\Delta\left(g_{1}, g_{2}\right)$ colors, then there exists a list supermodular coloring for $\left(g_{1}, g_{2}, L\right)$.

The aim of this paper is to provide a common generalization of Theorems 1.2 and 1.3 .

For an intersecting supermodular function $g: \mathcal{F} \rightarrow \mathbf{Z}$ on $U$, define the family $\mathcal{E}[g] \subseteq \mathcal{F}$ by

$$
\mathcal{E}[g]:=\left\{X \in \mathcal{F} \mid g(X) \geq 2, \quad \nexists X^{\prime} \in \mathcal{F}:\left[X^{\prime} \subsetneq X, g\left(X^{\prime}\right) \geq g(X)\right]\right\} .
$$

We call $\mathcal{E}[g]$ the effective set family of $g$. A function $d[g]: U \rightarrow \mathbf{N}$ is defined by

$$
d[g](u)=\max \{1, \max \{g(X) \mid u \in X \in \mathcal{E}[g]\}\},
$$

where $\max \{g(X) \mid u \in X \in \mathcal{E}[g]\}$ takes $-\infty$ if $\{X \mid u \in X \in \mathcal{E}[g]\}=\emptyset$.

Theorem 1.4 (Main Theorem). Let $g_{1}: \mathcal{F}_{1} \rightarrow \mathbf{Z}$ and $g_{2}: \mathcal{F}_{2} \rightarrow \mathbf{Z}$ be intersectingsupermodular functions such that $|X| \geq g_{i}(X)$ for every $i \in\{1,2\}$ and $X \in \mathcal{F}_{i}$. If each element $u \in U$ has a list $L(u)$ of $\max \left\{d\left[g_{1}\right](u), d\left[g_{2}\right](u)\right\}$ colors, then there exists a list supermodular coloring for $\left(g_{1}, g_{2}, L\right)$.

By definition, $\max \left\{d\left[g_{1}\right](u), d\left[g_{2}\right](u)\right\} \leq \Delta\left(g_{1}, g_{2}\right)$ for every $u \in U$, and hence Theorem 1.4 is an extension of Theorem 1.3. Also, Theorem 1.4 is a generalization of Theorem 1.2 as follows: For a bipartite graph $G=(S, T ; E)$, let $\mathcal{F}_{1}:=\left\{\delta_{G}(s) \mid s \in S\right\}$ and $\mathcal{F}_{2}:=\left\{\delta_{G}(t) \mid t \in T\right\}$, where $\delta_{G}(s) \subseteq E$ is the set of edges incident to $s$. Define $g_{1}: \mathcal{F}_{1} \rightarrow \mathbf{Z}$ by $g_{1}\left(\delta_{G}(s)\right)=\left|\delta_{G}(s)\right|$ and $g_{2}: \mathcal{F}_{2} \rightarrow \mathbf{Z}$ by $g_{2}\left(\delta_{G}(t)\right)=\left|\delta_{G}(t)\right|$. Then, $g_{1}, g_{2}$ are intersecting-supermodular functions on $E$. We see that an edge coloring of $G$ with $k$ colors is just a supermodular $k$-coloring for $\left(g_{1}, g_{2}\right)$. Moreover, for each edge $e=s t$, the value $\max \left\{d\left[g_{1}\right](e), d\left[g_{2}\right](e)\right\}$ coincides with $\max \left\{d_{G}(s), d_{G}(t)\right\}$. Thus, Theorem 1.4 generalizes both Theorems 1.2 and 1.3 .

Observe that, for every $u \in U$, the definition of $d\left[g_{i}\right]$ implies

$$
\max \left\{d\left[g_{1}\right](u), d\left[g_{2}\right](u)\right\} \leq \max \left\{1, \max \left\{g_{i}(X) \mid i \in\{1,2\}, u \in X \in \mathcal{F}_{i}\right\}\right\} .
$$

Theorem 1.4 then immediately implies the following corollary, which is weaker than Theorem 1.4 but can be stated without introducing $\mathcal{E}\left[g_{i}\right]$ nor $d\left[g_{i}\right]$.

Corollary 1.5. Let $g_{1}: \mathcal{F}_{1} \rightarrow \mathbf{Z}$ and $g_{2}: \mathcal{F}_{2} \rightarrow \mathbf{Z}$ be intersecting-supermodular functions such that $|X| \geq g_{i}(X)$ for every $i \in\{1,2\}$ and $X \in \mathcal{F}_{i}$. If each element $u \in U$ has a list $L(u)$ of $\max \left\{1, \max \left\{g_{i}(X) \mid i \in\{1,2\}, u \in X \in \mathcal{F}_{i}\right\}\right\}$ colors, then there exists a list supermodular coloring for $\left(g_{1}, g_{2}, L\right)$. 
Corollary 1.5 is properly weaker than Theorem 1.4 . (For example, let $g_{1}, g_{2}: \mathcal{F} \rightarrow \mathbf{Z}$ be the same function such that $\mathcal{F}=\left\{X^{\prime}, X\right\}, X^{\prime} \subsetneq X,\left|X^{\prime}\right| \geq 2$ and $g_{i}\left(X^{\prime}\right)=g_{i}(X)=2$. Then for an element $u \in X \backslash X^{\prime}$, we have $\max \left\{1, \max \left\{g_{i}(X) \mid i \in\{1,2\}, u \in X \in \mathcal{F}_{i}\right\}\right\}=2$ while $\max \left\{d\left[g_{1}\right](u), d\left[g_{2}\right](u)\right\}=1$.) However, we can see that Corollary 1.5 is still a common generalization of Theorems 1.2 and 1.3 .

We prove Theorem 1.4 by combining ideas of Iwata and Yokoi [6] and of Borodin et al. 2. By the result of Iwata and Yokoi (to be described in Proposition 2.1), we can reduce the problem of finding a list supermodular coloring to a problem of finding a pair of auxiliary functions satisfying certain conditions. We then show the existence of such auxiliary functions (Lemma 2.2), which is the main part of this paper. The proof of this lemma is by induction on the ground set. For that, we construct a special bipartite graph induced from the pair of intersecting-supermodular functions and apply a method (Proposition 4.1) used by Borodin et al. for bipartite edge coloring.

The rest of this paper is organized as follows. In Section 2, we introduce a key lemma, from which Theorem 1.4 follows. To prove this lemma, Section 3 introduces the notion of "bunch partitions" defined for intersecting-supermodular functions. There, we provide their properties, but proofs are postponed to Section 5 . Using bunch partitions, Section 4 shows the key lemma.

\section{Key Lemma}

In the proof of Theorem 1.3, Iwata and Yokoi proved the following proposition, which describes a sufficient condition for the existence of a list supermodular coloring in terms of two auxiliary functions $\pi_{1}$ and $\pi_{2}$.

Proposition 2.1 (Iwata and Yokoi [6, Proposition 3.2]). Let $g_{1}: \mathcal{F}_{1} \rightarrow \mathbf{Z}$ and $g_{2}: \mathcal{F}_{2} \rightarrow \mathbf{Z}$ be intersecting-supermodular functions. For an arbitrary function $f: U \rightarrow \mathbf{N}$, assume that there exist functions $\pi_{1}, \pi_{2}: U \rightarrow \mathbf{N}$ satisfying the following conditions.

(i) For every $u \in U$, we have $\pi_{1}(u)+\pi_{2}(u)-1 \leq f(u)$.

(ii) For each $i \in\{1,2\}, \pi_{i}$ dominates $g_{i}$.

Then, for any $L: U \rightarrow 2^{\Sigma}$ such that $|L(u)|=f(u)(\forall u \in U)$, there exists a list supermodular coloring for $\left(g_{1}, g_{2}, L\right)$.

Suppose that $\left(g_{1}, g_{2}, L\right)$ is provided. Proposition 2.1 says that, to show the existence of a list supermodular coloring, it suffices to find auxiliary functions $\pi_{1}, \pi_{2}$ satisfying conditions (i) and (ii) for $f$ such that $f(u)=|L(u)|$ for each $u \in U$. Indeed, Iwata and Yokoi proved Theorem 1.3 by showing the existence of such $\pi_{1}, \pi_{2}$ for the constant function $f=\Delta\left(g_{1}, g_{2}\right)$. In this case, the construction of $\pi_{1}$ and $\pi_{2}$ can be easily done by using Schrijver's result (see Remark 2.3).

In this paper, we deduce Theorem 1.4 from Proposition 2.1 by constructing $\pi_{1}$ and $\pi_{2}$ in a more careful manner. We show the following lemma.

Lemma 2.2 (Key Lemma). For any intersecting-supermodular functions $g_{1}: \mathcal{F}_{1} \rightarrow \mathbf{Z}$ and $g_{2}: \mathcal{F}_{2} \rightarrow \mathbf{Z}$ such that $|X| \geq g_{i}(X)$ for every $i \in\{1,2\}$ and $X \in \mathcal{F}_{i}$, there exist functions $\pi_{1}, \pi_{2}: U \rightarrow \mathbf{N}$ satisfying (i) and (ii) with $f(u)$ defined by $f(u)=\max \left\{d\left[g_{1}\right](u), d\left[g_{2}\right](u)\right\}$.

Once Lemma 2.2 is proved, we can immediately obtain Theorem 1.4 by combining it with Proposition 2.1. The remainder of this paper is devoted to showing Lemma 2.2.

Remark 2.3. For reference, we provide the method of Iwata and Yokoi [6] for a construction of $\pi_{1}$ and $\pi_{2}$ satisfying (i) and (ii) with $f$ defined by $f(u)=\Delta\left(g_{1}, g_{2}\right)$ for every $u \in U$. Let 
$k:=\Delta\left(g_{1}, g_{2}\right)$. Take a supermodular $k$-coloring $\pi: U \rightarrow[k]$ for $\left(g_{1}, g_{2}\right)$, whose existence is guaranteed by the result of Schrijver [8]. Define $\pi_{1}, \pi_{2}: U \rightarrow[k]$ by $\pi_{1}(u):=\pi(u)$ and $\pi_{2}(u):=k+1-\pi(u)$ for each $u \in U$. Then (i) holds because $\pi_{1}(u)+\pi_{2}(u)-1=k=f(u)$ for every $u$. Also (ii) holds as $g_{i}(X) \leq|\pi(X)|=\left|\pi_{1}(X)\right|=\left|\pi_{2}(X)\right|$ for any $i \in\{1,2\}$ and $X \in \mathcal{F}_{i}$.

\section{Bunch Partitions}

To show Lemma 2.2, this section introduces the notion of bunch partitions for intersectingsupermodular functions. This structure connects our supermodular coloring setting to a technique on bipartite graphs used by Borodin et al. [2].

Let $g: \mathcal{F} \rightarrow \mathbf{Z}$ be an intersecting-supermodular function on $U$. For a subset $K \subseteq U$, the reduction of $g$ by $K$ is the function $g_{K}: \mathcal{F}_{K} \rightarrow \mathbf{Z}$ defined by $\mathcal{F}_{K}=\{Z \backslash K \mid Z \in \mathcal{F}\}$ and

$$
g_{K}(X)=\max \left\{\hat{g}_{K}(Z) \mid Z \in \mathcal{F}, Z \backslash K=X\right\} \quad\left(X \in \mathcal{F}_{K}\right),
$$

where $\hat{g}_{K}(Z)=g(Z)-1$ for $Z \in \mathcal{F}$ with $Z \cap K \neq \emptyset$ and $\hat{g}_{K}(Z)=g(Z)$ for $Z \in \mathcal{F}$ with $Z \cap K=\emptyset$. The following fact is known (cf., Frank [3], Iwata and Yokoi [6, Claim 2.1]).

Claim 3.1. The reduction $g_{K}: \mathcal{F}_{K} \rightarrow \mathbf{Z}$ is an intersecting-supermodular function.

Recall that the effective set family $\mathcal{E}[g]$ is a collection of subsets $X \in \mathcal{F}$ such that $g(X) \geq 2$ and no proper subset $X^{\prime} \in \mathcal{F}$ satisfies $g\left(X^{\prime}\right) \geq g(X)$. From $\mathcal{E}[g]$, define a family $\mathcal{P}[g]$ by

$$
\mathcal{P}[g]:=\left\{X \in \mathcal{E}[g] \mid \nexists X^{\prime} \in \mathcal{E}[g]: X \subsetneq X^{\prime}\right\} \cup\{\{u\} \mid u \in U \backslash \bigcup \mathcal{E}[g]\} .
$$

That is, $\mathcal{P}[g]$ contains all maximal members of $\mathcal{E}[g]$ and singleton sets of elements not included in any member of $\mathcal{E}[g]$ (see Figure 1 for an example). From the definitions of $\mathcal{E}[g]$ and $\mathcal{P}[g]$, we can obtain the following two claims. The first is clear by definition.

Claim 3.2. For any $X \in \mathcal{E}[g]$, there exists $P \in \mathcal{P}[g] \cap \mathcal{E}[g]$ such that $X \subseteq P$.

Claim 3.3. For any $X \in \mathcal{F}$ such that $X \notin \mathcal{E}[g]$ and $g(X) \geq 2$, there exist $X^{\prime} \in \mathcal{E}[g]$ and $P \in \mathcal{P}[g] \cap \mathcal{E}[g]$ such that $X^{\prime} \subseteq X \cap P$ and $g\left(X^{\prime}\right) \geq g(X)$.

Proof. For such $X$, let $X^{\prime}$ be a minimal maximizer of $g$ subject to $X^{\prime} \subseteq X$. Then, $X \notin \mathcal{E}[g]$ implies $X^{\prime} \subsetneq X$ and $g\left(X^{\prime}\right) \geq g(X) \geq 2$. By definition, $X^{\prime}$ belongs to $\mathcal{E}[g]$. Then, by Claim 3.2 . there is $P \in \mathcal{P}[g] \cap \mathcal{E}[g]$ with $X^{\prime} \subseteq P$. They satisfy $X^{\prime} \subseteq X \cap P$ and $g\left(X^{\prime}\right) \geq g(X)$.

We provide the following four properties of $\mathcal{P}[g]$, whose proofs are postponed to Section 5 .

Proposition 3.4. The family $\mathcal{P}[g]$ is a partition of $U$.

We call $\mathcal{P}[g]$ the bunch partition of $U$ by $g$. We denote by $P[g](u)$ the unique part containing $u \in U$. Recall that $d[g]$ is defined by $d[g](u)=\max \{1, \max \{g(X) \mid u \in X \in \mathcal{E}[g]\}\}$.

Proposition 3.5. For every $u \in \bigcup \mathcal{E}[g]$, we have $P[g](u) \in \mathcal{E}[g]$ and $d[g](u)=g(P[g](u)) \geq 2$. For every $u \in U \backslash \bigcup \mathcal{E}[g]$, we have $P[g](u)=\{u\}$ and $d[g](u)=1$.

A subset $K \subseteq U$ is called a partial transversal of $\mathcal{P}[g]$ if $|K \cap P| \leq 1$ for every $P \in \mathcal{P}[g]$.

Proposition 3.6. Suppose $|Z| \geq g(Z)$ for every $Z \in \mathcal{F}$. Then, for any partial transversal $K$ of $\mathcal{P}[g]$, the reduction $g_{K}$ satisfies $|X| \geq g_{K}(X)$ for every $X \in \mathcal{F}_{K}$.

Proposition 3.7. Suppose $|Z| \geq g(Z)$ for every $Z \in \mathcal{F}$ and take an arbitrary partial transversal $K$ of $\mathcal{P}[g]$. For every $u \in U \backslash K$, we see that $P[g](u) \cap K=\emptyset$ implies $d\left[g_{K}\right](u)=d[g](u)$ and $P[g](u) \cap K \neq \emptyset$ implies $d\left[g_{K}\right](u)<d[g](u)$. 


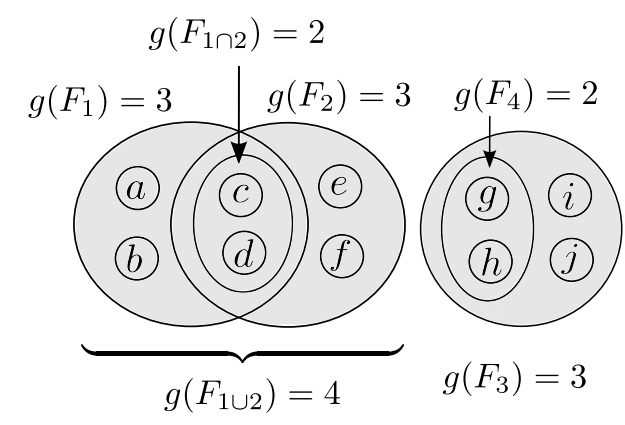

(1) Before reduction

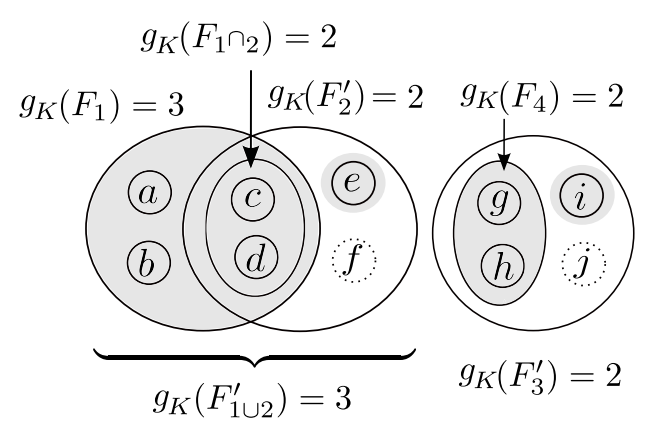

(2) After reduction by $K=\{f, j\}$

Figure 1: (1) The family $\mathcal{F}$ in Example 3.8 and values of $g$. (2) The family $\mathcal{F}_{K}$ and values of $g_{K}$, where $F^{\prime}$ denotes $F \backslash K$ for each $F \in \mathcal{F}$. The families $\mathcal{P}[k]$ and $\mathcal{P}\left[g_{K}\right]$ are represented by gray color.

To capture the notion of bunch partitions, we provide an example.

Example 3.8. Let $U=\{a, b, c, d, e, f, g, h, i, j\}$ and $\mathcal{F}=\left\{F_{1}, F_{2}, F_{1 \cup 2}, F_{1 \cap 2}, F_{3}, F_{4}\right\}$, where $F_{1}=\{a, b, c, d\}, F_{2}=\{c, d, e, f\}, F_{1 \cup 2}=F_{1} \cup F_{2}, F_{1 \cap 2}=F_{1} \cap F_{2}, F_{3}=\{g, h, i, j\}, F_{4}=\{g, h\}$. Define $g: \mathcal{F} \rightarrow \mathbf{Z}$ by $g\left(F_{1}\right)=g\left(F_{2}\right)=g\left(F_{3}\right)=3, g\left(F_{1 \cup 2}\right)=4, g\left(F_{1 \cap 2}\right)=g\left(F_{4}\right)=2$ (see Figure 11). Then, $\mathcal{E}[g]=\mathcal{F}, \mathcal{P}[g]=\left\{F_{1 \cup 2}, F_{3}\right\}$. Also, $d[g](u)=4$ for each $u \in F_{1 \cup 2}$ and $d[g](u)=3$ for each $u \in F_{3}$.

Let $K=\{f, j\}$ and $g_{K}$ be the reduction of $g$ by $K$. Then, $\mathcal{E}\left[g_{K}\right]=\left\{F_{1}, F_{1 \cap 2}, F_{4}\right\}$ and $\mathcal{P}[g]=\left\{F_{1},\{e\}, F_{4},\{i\}\right\}$, Also, $d[g](u)=3$ for each $u \in F_{1}, d[g](u)=2$ for each $u \in F_{4}$, and $d[g](e)=d[g](i)=1$.

Remark 3.9. The definition of a bunch partition is similar to that of a solid partition studied by Bárász, Becker, and Frank [1]. For a directed graph, a vertex set $X$ is called in-solid if there is no nonempty proper subset $X^{\prime}$ of $X$ satisfying $\varrho\left(X^{\prime}\right) \leq \varrho(X)$, where $\varrho(X)$ is the in-degree, i.e., the number of edges entering $X$. Using the submodularity of $\varrho$, it was shown in [1] that maximal in-solid sets form a partition. (The same is true for out-solid sets, which are defined analogously by the out-degrees.) Proposition 3.4 will be shown by similar arguments (in Section 5).

\section{Proof of the Key Lemma}

This section proves Lemma 2.2 relying on Propositions 3.4 3.7. (In fact, we prove a stronger version of Lemma 2.2.) First, we introduce the following fact on bipartite graphs, which was used by Borodin et al. 2] to show Theorem 1.2. For completeness, we provide their proof.

Proposition 4.1 (Borodin et al. [2, Lemma 3.1]). For a bipartite graph $G=(S, T ; E)$, if $|S| \geq|T|$ and $S$ contains no isolated vertices, then $G$ has a nonempty matching $M \subseteq E$ such that every $e=s t \in E$ with $s \in \partial M$ satisfies $t \in \partial M$, where $\partial M \subseteq S \cup T$ is the set of vertices incident to some edge in $M$.

Proof [2]. Since $|T| \leq|S|$, we have $|\Gamma(S)| \leq|T| \leq|S|$, where $\Gamma(S)$ denotes the set of neighbors of vertices in $S$. Let $V$ be a minimal nonempty subset of $S$ such that $|\Gamma(V)| \leq|V|$. Then $|\Gamma(V)|=|V|$ and there is a matching $M$ with $\partial M \cap S=V$. (If $|V|=1$, this holds because $S$ contains no isolated vertices. If $|V| \geq 2$, the minimality of $V$ implies $|\Gamma(V)|=|V|$ and $|\Gamma(W)|>|W|$ whenever $\emptyset \subsetneq W \subsetneq V$, and hence the König-Hall theorem implies that there is a matching $M$ with $\partial M \cap S=V$.) By $\partial M \cap T \subseteq \Gamma(V)$ and $|\partial M \cap T|=|\partial M \cap S|=|V|=|\Gamma(V)|$, we obtain $\partial M \cap T=\Gamma(V)$. We see that this $M$ has the required property. 
For the pair of partitions of the same ground set, Proposition 4.1 is rephrased as follows.

Claim 4.2. Let $g_{1}: \mathcal{F}_{1} \rightarrow \mathbf{Z}$ and $g_{2}: \mathcal{F}_{2} \rightarrow \mathbf{Z}$ be intersecting-supermodular functions. For the bunch partitions $\mathcal{P}\left[g_{1}\right]$ and $\mathcal{P}\left[g_{2}\right]$, there exists a nonempty common partial transversal $K \subseteq U$ satisfying one or both of the following.

(a) Every $u \in U$ with $P\left[g_{1}\right](u) \cap K \neq \emptyset$ satisfies $P\left[g_{2}\right](u) \cap K \neq \emptyset$.

(b) Every $u \in U$ with $P\left[g_{2}\right](u) \cap K \neq \emptyset$ satisfies $P\left[g_{1}\right](u) \cap K \neq \emptyset$.

Proof. Let us denote the bunch partitions $\mathcal{P}\left[g_{1}\right]$ and $\mathcal{P}\left[g_{2}\right]$ by

$$
\mathcal{P}\left[g_{1}\right]=\left\{P_{1}^{1}, P_{2}^{1}, \ldots, P_{q_{1}}^{1}\right\}, \quad \mathcal{P}\left[g_{2}\right]=\left\{P_{1}^{2}, P_{2}^{2}, \ldots, P_{q_{2}}^{2}\right\} .
$$

Let $G^{\prime}=\left(S^{\prime}, T^{\prime} ; E^{\prime}\right)$ be a bipartite graph such that each vertex corresponds to a part of partitions and each edge corresponds to an element. That is, we define

$$
\begin{aligned}
& S^{\prime}=\left\{s_{j} \mid j=1,2, \ldots, q_{1}\right\}, \quad T^{\prime}=\left\{t_{k} \mid k=1,2, \ldots, q_{2}\right\}, \\
& E^{\prime}=\left\{e_{u}=s_{j} t_{k} \mid u \in U, P\left[g_{1}\right](u)=P_{j}^{1}, P\left[g_{2}\right](u)=P_{k}^{2}\right\} .
\end{aligned}
$$

There is a one-to-one correspondence between $U$ and $E^{\prime}$. Clearly, $G^{\prime}$ has no isolated vertex.

We first consider the case $\left|S^{\prime}\right| \geq\left|T^{\prime}\right|$. In this case, apply Proposition 4.1 with $S=S^{\prime}$ and $T=T^{\prime}$. Then, there is a nonempty matching $M \subseteq E^{\prime}$ such that every $e_{u}=s_{j} t_{k} \in E^{\prime}$ with $s_{j} \in \partial M$ satisfies $t_{k} \in \partial M$. Let $K:=\left\{u \in U \mid e_{u} \in M\right\}$. As $M$ is a nonempty matching, $K$ is a nonempty common partial transversal of $\mathcal{P}\left[g_{1}\right]$ and $\mathcal{P}\left[g_{2}\right]$. Also, the condition of $M$ means that every $u \in U$ with $P\left[g_{1}\right](u) \cap K \neq \emptyset$ satisfies $P\left[g_{2}\right](u) \cap K \neq \emptyset$. Thus, (a) holds.

In the case $\left|S^{\prime}\right|<\left|T^{\prime}\right|$, by applying Proposition 4.1 with $S=T^{\prime}$ and $T=S^{\prime}$, we can similarly obtain a nonempty common partial transversal $K$ satisfying (b).

We are now ready to prove Lemma 2.2. Actually, we show the following stronger statement, in which an additional constraint (iii) is also required for the functions $\pi_{1}$ and $\pi_{2}$. It is clear that Lemma 4.3 implies Lemma 2.2 .

Lemma 4.3 (Stronger Version of Lemma 2.2). For any intersecting-supermodular functions $g_{1}: \mathcal{F}_{1} \rightarrow \mathbf{Z}$ and $g_{2}: \mathcal{F}_{2} \rightarrow \mathbf{Z}$ such that $|X| \geq g_{i}(X)$ for every $i \in\{1,2\}$ and $X \in \mathcal{F}_{i}$, there exist functions $\pi_{1}, \pi_{2}: U \rightarrow \mathbf{N}$ satisfying the following (i), (ii), and (iii).

(i) For every $u \in U$, we have $\pi_{1}(u)+\pi_{2}(u)-1 \leq \max \left\{d\left[g_{1}\right](u), d\left[g_{2}\right](u)\right\}$.

(ii) For each $i \in\{1,2\}, \pi_{i}$ dominates $g_{i}$.

(iii) For each $i \in\{1,2\}$ and $u \in U$, we have $\pi_{i}(u) \leq d\left[g_{i}\right](u)$.

Proof of Lemma 4.3 (and hence of Lemma 2.2). We use induction on $|U|$, i.e., the size of the ground set. Recall that $d\left[g_{i}\right](u)=\max \left\{1, \max \left\{g_{i}(Z) \mid u \in Z \in \mathcal{E}\left[g_{i}\right]\right\}\right\}$.

First, consider the case $|U|=1$, i.e., $U=\{u\}$. Since $g_{i}(Z) \leq|Z| \leq 1$ for every $i \in\{1,2\}$ and $X \in \mathcal{F}_{i}$, we have $d\left[g_{1}\right](u)=d\left[g_{2}\right](u)=1$. Let $\pi_{1}(u)=\pi_{2}(u)=1$. Then, we can observe that (i), (ii), and (iii) are all satisfied.

We now consider the case $|U|>1$. By Claim 4.2 , there is a nonempty common partial transversal $K$ of $\mathcal{P}\left[g_{1}\right]$ and $\mathcal{P}\left[g_{2}\right]$ satisfying (a) or (b). For each $i \in\{1,2\}$, we denote by $g_{i}^{\prime}$ the reduction of $g_{i}$ by $K$. The domain of $g_{i}^{\prime}$ is denoted by $\mathcal{F}_{i}^{\prime}=\left\{Z \backslash K \mid Z \in \mathcal{F}_{i}\right\}$. By Claim 3.1 and Proposition 3.6, $g_{1}^{\prime}$ and $g_{2}^{\prime}$ are intersecting-supermodular functions on $U \backslash K$ satisfying $|X| \geq g_{i}^{\prime}(X)$ for every $i \in\{1,2\}$ and $X \in \mathcal{F}_{i}^{\prime}$. Since $|U \backslash K|<|U|$, the inductive 
assumption implies that there exist $\pi_{1}^{\prime}, \pi_{2}^{\prime}: U \backslash K \rightarrow \mathbf{N}$ such that (i), (ii), and (iii) hold with $\left(U \backslash K, \pi_{1}^{\prime}, \pi_{2}^{\prime}, g_{1}^{\prime}, g_{2}^{\prime}\right)$ in place of $\left(U, \pi_{1}, \pi_{2}, g_{1}, g_{2}\right)$. By (i) and (iii), for every $u \in U \backslash K$, we have

$$
\begin{aligned}
& \pi_{1}^{\prime}(u)+\pi_{2}^{\prime}(u)-1 \leq \max \left\{d\left[g_{1}^{\prime}\right](u), d\left[g_{2}^{\prime}\right](u)\right\} \\
& \pi_{i}^{\prime}(u) \leq d\left[g_{i}^{\prime}\right](u) \quad(i \in\{1,2\}) .
\end{aligned}
$$

By (ii), for each $i \in\{1,2\}$, we have

$$
\left|\pi_{i}^{\prime}(X)\right| \geq g_{i}^{\prime}(X) \quad\left(X \in \mathcal{F}_{i}^{\prime}\right) .
$$

By the definition of the reduction $g_{i}^{\prime}$, for each $i \in\{1,2\}$, we have

$$
\begin{aligned}
g_{i}^{\prime}(Z) & \geq g_{i}(Z) & & \left(Z \in \mathcal{F}_{i}, Z \cap K=\emptyset\right), \\
g_{i}^{\prime}(Z \backslash K) & \geq g_{i}(Z)-1 & & \left(Z \in \mathcal{F}_{i}, Z \cap K \neq \emptyset\right) .
\end{aligned}
$$

Also, since $K$ is a common partial transversal, Proposition 3.7 implies that, for every $i \in\{1,2\}$ and $u \in U \backslash K$, we have

$$
\begin{gathered}
d\left[g_{i}^{\prime}\right](u) \leq d\left[g_{i}\right](u), \\
P\left[g_{i}\right](u) \cap K \neq \emptyset \Longrightarrow d\left[g_{i}^{\prime}\right](u)<d\left[g_{i}\right](u) .
\end{gathered}
$$

Recall that $K$ satisfies (a) or (b). We now show the case in which (a) holds (the case for (b) is shown analogously). Then, for every $u \in U$, we have

$$
P\left[g_{1}\right](u) \cap K \neq \emptyset \Longrightarrow P\left[g_{2}\right](u) \cap K \neq \emptyset .
$$

Using the functions $\pi_{1}^{\prime}, \pi_{2}^{\prime}: U \backslash K \rightarrow \mathbf{N}$, we define $\pi_{1}, \pi_{2}: U \rightarrow \mathbf{N}$ by

$$
\begin{aligned}
& \pi_{1}(u)= \begin{cases}1 & (u \in K), \\
\pi_{1}^{\prime}(u) & \left(u \in U \backslash K, P\left[g_{1}\right](u) \cap K=\emptyset\right), \\
\pi_{1}^{\prime}(u)+1 & \left(u \in U \backslash K, P\left[g_{1}\right](u) \cap K \neq \emptyset\right),\end{cases} \\
& \pi_{2}(u)= \begin{cases}d\left[g_{2}\right](u) & (u \in K), \\
\pi_{2}^{\prime}(u) & (u \in U \backslash K) .\end{cases}
\end{aligned}
$$

We check that these $\pi_{1}$ and $\pi_{2}$ satisfy conditions (i), (ii), and (iii).

Conditions (i) and (iii): There are three cases corresponding to the definition of $\pi_{1}(u)$.

If $u \in K$, then we have $\pi_{1}(u)=1$ and $\pi_{2}(u)=d\left[g_{2}\right](u)$. Then, $\pi_{1}(u)+\pi_{2}(u)-1=d\left[g_{2}\right](u) \leq$ $\max \left\{d\left[g_{1}\right](u), d\left[g_{2}\right](u)\right\}$. Also, $\pi_{i}(u) \leq d\left[g_{i}\right](u)$ for each $i \in\{1,2\}$.

If $u \in U \backslash K$ and $P\left[g_{1}\right](u) \cap K=\emptyset$, then $\pi_{1}(u)=\pi_{1}^{\prime}(u)$ and $\pi_{2}(u)=\pi_{2}^{\prime}(u)$. By (4.1) and (4.6), we have $\pi_{1}(u)+\pi_{2}(u)-1=\pi_{1}^{\prime}(u)+\pi_{2}^{\prime}(u)-1 \leq \max \left\{d\left[g_{1}^{\prime}\right](u), d\left[g_{2}^{\prime}\right](u)\right\} \leq$ $\max \left\{d\left[g_{1}\right](u), d\left[g_{2}\right](u)\right\}$. Also, 4.2) and (4.6) imply $\pi_{i}(u) \leq d\left[g_{i}\right](u)$ for each $i \in\{1,2\}$.

If $u \in U \backslash K$ and $P\left[g_{1}\right](u) \cap K \neq \emptyset$, then $\pi_{1}(u)=\pi_{1}^{\prime}(u)+1$ and $\pi_{2}(u)=\pi_{2}^{\prime}(u)$. From 4.8 and (4.7), we have $d\left[g_{1}^{\prime}\right](u)<d\left[g_{1}\right](u)$ and $d\left[g_{2}^{\prime}\right](u)<d\left[g_{2}\right](u)$, and hence

$$
\max \left\{d\left[g_{1}^{\prime}\right](u), d\left[g_{2}^{\prime}\right](u)\right\}<\max \left\{d\left[g_{1}\right](u), d\left[g_{2}\right](u)\right\} .
$$

With 4.1), this implies $\pi_{1}(u)+\pi_{2}(u)-1=\left(\pi_{1}^{\prime}(u)+\pi_{2}^{\prime}(u)-1\right)+1 \leq \max \left\{d\left[g_{1}^{\prime}\right](u), d\left[g_{2}^{\prime}\right](u)\right\}+1 \leq$ $\max \left\{d\left[g_{1}\right](u), d\left[g_{2}\right](u)\right\}$. Also, 4.2$)$ and $d\left[g_{i}^{\prime}\right](u)<d\left[g_{i}\right](u)$ imply $\pi_{i}(u) \leq d\left[g_{i}\right](u)$ for each $i$. 
Condition (ii): We show $\left|\pi_{i}(Z)\right| \geq g_{i}(Z)$ for any $i \in\{1,2\}$ and $Z \in \mathcal{F}_{i}$. If $g_{i}(Z) \leq 0$, the claim is clear. Also, if $g_{i}(Z)=1$, then the assumption $|Z| \geq g_{i}(Z)$ implies $Z \neq \emptyset$ and hence $\left|\pi_{i}(Z)\right| \geq 1=g_{i}(Z)$. Therefore, let us assume $g_{i}(Z) \geq 2$.

By Claims 3.2 and 3.3 , then there exist $\hat{Z} \in \mathcal{E}\left[g_{i}\right]$ and $\hat{P} \in \mathcal{P}\left[g_{i}\right] \cap \mathcal{E}\left[g_{i}\right]$ satisfying

$$
\hat{Z} \subseteq Z, \quad \hat{Z} \subseteq \hat{P}, \quad g_{i}(\hat{Z}) \geq g_{i}(Z)
$$

(In particular, $\hat{Z}=Z$ if $Z \in \mathcal{E}\left[g_{i}\right]$.) We need to consider cases $i=1$ and $i=2$ separately.

Case $i=1$. Note that $\hat{P}$ in $(4.9)$ satisfies $\hat{P}=P\left[g_{1}\right](u)$ for all $u \in \hat{Z} \subseteq \hat{P}$. Therefore, by the definition of $\pi_{1}$, if $\hat{P} \cap K=\emptyset$, then $\pi_{1}(u)=\pi_{1}^{\prime}(u)$ for all $u \in \hat{Z} \backslash K$. Also, if $\hat{P} \cap K \neq \emptyset$, then $\pi_{1}(u)=\pi_{1}^{\prime}(u)+1$ for all $u \in \hat{Z} \backslash K$. Thus, in both cases, we have

$$
\left|\pi_{1}(\hat{Z} \backslash K)\right|=\left|\pi_{1}^{\prime}(\hat{Z} \backslash K)\right| .
$$

If $\hat{Z} \cap K=\emptyset$, then 4.10 means $\left|\pi_{1}(\hat{Z})\right|=\left|\pi_{1}^{\prime}(\hat{Z})\right|$. With (4.3), (4.4), (4.9), this implies the following inequality. (Note that the monotonicity of $\left|\pi_{1}(\cdot)\right|$ and $\hat{Z} \subseteq Z$ imply $\left|\pi_{1}(\hat{Z})\right| \leq\left|\pi_{1}(Z)\right|$.)

$$
\left|\pi_{1}(Z)\right| \geq\left|\pi_{1}(\hat{Z})\right|=\left|\pi_{1}^{\prime}(\hat{Z})\right| \geq g_{1}^{\prime}(\hat{Z}) \geq g_{1}(\hat{Z}) \geq g_{1}(Z) .
$$

If $\hat{Z} \cap K \neq \emptyset$, then, as mentioned above, $\pi_{1}(u)=\pi_{1}^{\prime}(u)+1>1$ for all $u \in \hat{Z} \backslash K$. This implies $1 \notin \pi_{1}(\hat{Z} \backslash K)$. Since $\pi_{1}(u)=1$ for any $u \in \hat{Z} \cap K \neq \emptyset$, we have $\left|\pi_{1}(\hat{Z})\right|=\left|\pi_{1}(\hat{Z} \backslash K)\right|+1$. With 4.3, 4.5), 4.9), 4.10, this implies

$$
\left|\pi_{1}(Z)\right| \geq\left|\pi_{1}(\hat{Z})\right|=\left|\pi_{1}(\hat{Z} \backslash K)\right|+1=\left|\pi_{1}^{\prime}(\hat{Z} \backslash K)\right|+1 \geq g_{1}^{\prime}(\hat{Z} \backslash K)+1 \geq g_{1}(\hat{Z}) \geq g_{1}(Z) .
$$

Case $i=2$. By the definition of $\pi_{2}$, we have $\pi_{2}(u)=\pi_{2}^{\prime}(u)$ for every $u \in U \backslash K$. Hence,

$$
\pi_{2}(\hat{Z} \backslash K)=\pi_{2}^{\prime}(\hat{Z} \backslash K) .
$$

If $\hat{Z} \cap K=\emptyset$, then (4.11) implies $\left|\pi_{2}(\hat{Z})\right|=\left|\pi_{2}^{\prime}(\hat{Z})\right|$. With (4.3), 4.4), (4.9), this implies

$$
\left|\pi_{2}(Z)\right| \geq\left|\pi_{2}(\hat{Z})\right|=\left|\pi_{2}^{\prime}(\hat{Z})\right| \geq g_{2}^{\prime}(\hat{Z}) \geq g_{2}(\hat{Z}) \geq g_{2}(Z) .
$$

If $\hat{Z} \cap K \neq \emptyset$, then $\hat{P}$ in 4.9 satisfies $\hat{Z} \cap K \subseteq \hat{P} \cap K \neq \emptyset$. For every $u \in \hat{Z} \backslash K \subseteq \hat{P}$, since $P\left[g_{2}\right](u)=\hat{P}$ holds, 4.7$)$ implies $d\left[g_{2}^{\prime}\right](u)<d\left[g_{2}\right](u)$. Also, $d\left[g_{2}\right](u)=g_{2}(\hat{P})$ by Proposition 3.5 . By (4.2), then every $u \in \hat{Z} \backslash K$ satisfies

$$
\pi_{2}(u)=\pi_{2}^{\prime}(u) \leq d\left[g_{2}^{\prime}\right](u)<d\left[g_{2}\right](u)=g_{2}(\hat{P}),
$$

and hence $g_{2}(\hat{P}) \notin \pi_{2}(\hat{Z} \backslash K)$. Since any $\hat{u} \in \hat{Z} \cap K \subseteq \hat{P}$ satisfies $\pi_{2}(\hat{u})=d\left[g_{2}\right](\hat{u})=g_{2}(\hat{P})$ by the definition of $\pi_{2}$, this implies $\left|\pi_{2}(\hat{Z})\right|=\left|\pi_{2}(\hat{Z} \backslash K)\right|+1$. By (4.3), (4.5), (4.9), (4.11), then

$$
\left|\pi_{2}(Z)\right| \geq\left|\pi_{2}(\hat{Z})\right|=\left|\pi_{2}(\hat{Z} \backslash K)\right|+1 \geq\left|\pi_{2}^{\prime}(\hat{Z} \backslash K)\right|+1 \geq g_{2}^{\prime}(\hat{Z} \backslash K)+1 \geq g_{2}(\hat{Z}) \geq g_{2}(Z)
$$

follows.

\section{$5 \quad$ Properties of Bunch Partitions}

This section shows Propositions 3.4 3.7, which state properties of bunch partitions. The first subsection gives some basic properties and proves Propositions 3.4 and 3.5 . The second shows properties related to reduction by partial transversals and proves Propositions 3.6 and 3.7 . 


\subsection{Basic Properties}

Let $g: \mathcal{F} \rightarrow \mathbf{Z}$ be an intersecting-supermodular function on $U$. Recall that the effective set family $\mathcal{E}[g]$ is defined as the family of subsets $X \in \mathcal{F}$ satisfying

$$
\begin{aligned}
& g(X) \geq 2 \\
& \nexists X^{\prime} \in \mathcal{F}:\left[X^{\prime} \subsetneq X, g\left(X^{\prime}\right) \geq g(X)\right] .
\end{aligned}
$$

Claim 5.1. If $X, Y \in \mathcal{E}[g]$ are intersecting, $g(X \cup Y)>\max \{g(X), g(Y)\}$ and $X \cup Y \in \mathcal{E}[g]$.

Proof. As $X, Y \in \mathcal{E}[g]$ are intersecting, we have $g(X)+g(Y) \leq g(X \cup Y)+g(X \cap Y)$. Also, as $X \cap Y \subsetneq X$, (5.2) for $X$ implies $g(X)>g(X \cap Y)$. Thus, we obtain $g(Y)<g(X \cap Y)$. Similarly, from (5.2) for $Y$, we obtain $g(X)<g(X \cap Y)$. Thus, $g(X \cup Y)>\max \{g(X), g(Y)\} \geq 2$.

Let $Z$ be a minimal maximizer of $g$ in $\left\{Z^{\prime} \mid Z^{\prime} \in \mathcal{F}, Z^{\prime} \subseteq X \cup Y\right\}$. To show $X \cup Y \in \mathcal{E}[g]$, it suffices to prove $Z=X \cup Y$. Suppose, to the contrary, we have $Z \subsetneq X \cup Y$, which implies either $X \backslash Z \neq \emptyset$ or $Y \backslash Z \neq \emptyset$. Without loss of generality, let $X \backslash Z \neq \emptyset$. By definition, $Z$ satisfies $g(Z) \geq g(X \cup Y)>\max \{g(X), g(Y)\}$. This implies $Z \nsubseteq X$ and $Z \nsubseteq Y$ since $X$ and $Y$ satisfy (5.2). Combining $Z \subseteq X \cup Y$ and $Z \nsubseteq Y$ implies $X \cap Z \neq \emptyset$. Thus, we have

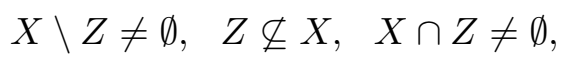

which mean that $X$ and $Z$ are intersecting. Then, $g(X)+g(Z) \leq g(X \cup Z)+g(X \cap Z)$. As $g(X)>g(X \cap Z)$ by $(5.2)$ for $X$, we obtain $g(Z)<g(X \cup Z)$, which contradicts the fact that $Z$ is a maximizer.

Recall that $\mathcal{P}[g]$ consists of all maximal members of $\mathcal{E}[g]$ and the singleton sets $\{u\}$ of all $u \in U \backslash \bigcup \mathcal{E}[g]$. Then, Claim 5.1 implies Proposition 3.4 as follows.

Proof of Proposition 3.4. We show that $\mathcal{P}[g]$ is a partition. By the definition of $\mathcal{P}[g]$, it suffices to show that the maximal members of $\mathcal{E}[g]$ are all pairwise disjoint. Suppose, to the contrary, that distinct $X, Y \in \mathcal{E}[g]$ are maximal and not disjoint. Then, they are intersecting, and Claim 5.1 implies $X \cup Y \in \mathcal{E}[g]$, which contradicts the maximality of $X$ and $Y$.

Recall that $d[g]: U \rightarrow \mathbf{N}$ is defined by $d[g](u)=\max \{1, \max \{g(X) \mid u \in X \in \mathcal{E}[g]\}\}$. Also, recall that $P[g](u)$ is defined as the unique part of $\mathcal{P}[g]$ containing $u$.

Proof of Proposition 3.5. If $u \in \bigcup \mathcal{E}[g]$, then $P[g](u)$ is a maximal member of $\mathcal{E}[g]$. Since all maximal members of $\mathcal{E}[g]$ are pairwise disjoint by Proposition 3.4 any $X \in \mathcal{E}[g]$ with $X \cap P[g](u) \neq \emptyset$ satisfies $X \subseteq P[g](u)$. Because $u \in P[g](u)$, then the condition $u \in X \in \mathcal{E}[g]$ implies $X \subseteq P[g](u)$, from which $g(X) \leq g(P[g](u))$ follows because $P[g](u) \in \mathcal{E}[g]$. Thus, $d[g](u)=\max \{g(X) \mid u \in X \in \mathcal{E}[g]\}=g(P[g](u))$. Also $g(P[g](u)) \geq 2$ by $P[g](u) \in \mathcal{E}[g]$.

If $u \notin \cup \mathcal{E}[g]$, then the claim immediately follows from the definitions of $\mathcal{P}[g]$ and $d[g]$.

\subsection{Reduction by a Partial Transversal}

As before, let $g: \mathcal{F} \rightarrow \mathbf{Z}$ be an intersecting-supermodular function. We also assume

$$
|Z| \geq g(Z) \quad(Z \in \mathcal{F}) .
$$

Under this assumption, 5.1) implies the following observation.

Claim 5.2. Every $Z \in \mathcal{E}[g]$ satisfies $|Z| \geq 2$.

\footnotetext{
${ }^{2}$ In contrast, $\mathcal{E}[g]$ is not closed under taking intersection.
} 
Take a partial transversal $K$ of $\mathcal{P}[g]$ and let $g_{K}: \mathcal{F}_{K} \rightarrow \mathbf{Z}$ be the reduction of $g$ by $K$, i.e.,

$$
g_{K}(X)=\max \left\{\hat{g}_{K}(Z) \mid Z \in \mathcal{F}, Z \backslash K=X\right\},
$$

where $\hat{g}_{K}(Z)=g(Z)-1$ for $Z \in \mathcal{F}$ with $Z \cap K \neq \emptyset$ and $\hat{g}_{K}(Z)=g(Z)$ for $Z \in \mathcal{F}$ with $Z \cap K=\emptyset$. We often use the following observation.

Claim 5.3. For any $Z^{\prime}, Z \in \mathcal{F}$, we have

$$
\begin{aligned}
{\left[Z^{\prime} \subseteq Z, g\left(Z^{\prime}\right) \geq g(Z)\right] } & \Longrightarrow \hat{g}_{K}\left(Z^{\prime}\right) \geq \hat{g}_{K}(Z), \\
g\left(Z^{\prime}\right)>g(Z) & \Longrightarrow \hat{g}_{K}\left(Z^{\prime}\right) \geq \hat{g}_{K}(Z) .
\end{aligned}
$$

Proof. For (5.5), observe that $Z^{\prime} \subseteq Z$ implies $\left[Z^{\prime} \cap K \neq \emptyset \Longrightarrow Z \cap K \neq \emptyset\right]$. (5.6) is clear.

Proof of Proposition 3.6. We show $|X| \geq g_{K}(X)$ for any $X \in \mathcal{F}_{K}$. By the definition of $g_{K}$, it suffices to show $|Z \backslash K| \geq \hat{g}_{K}(Z)$ for every $Z \in \mathcal{F}$. If $Z \cap K=\emptyset$, then (5.3) immediately implies $|Z \backslash K|=|Z| \geq g(Z)=\hat{g}_{K}(Z)$. Also, if $Z \cap K \neq \emptyset$ and $g(Z) \leq 1$, then $\hat{g}_{K}(Z) \leq 0$, and hence clearly $|Z \backslash K| \geq \hat{g}_{K}(Z)$. Therefore, we assume $Z \cap K \neq \emptyset$ and $g(Z) \geq 2$.

In the case $Z \in \mathcal{E}[g]$, by Claim 3.2 , there is $P \in \mathcal{P}[g] \cap \mathcal{E}[g]$ with $Z \subseteq P$. Since $K$ is a partial transversal of $\mathcal{P}[g]$, we have $|Z \cap K| \leq|P \cap K| \leq 1$. By (5.3) and $Z \cap K \neq \emptyset$, then $|Z \backslash K|=|Z|-1 \geq g(Z)-1=\hat{g}_{K}(Z)$.

In the case $Z \notin \mathcal{E}[g]$, Claim 3.3 implies that some $Z^{\prime} \in \mathcal{F}$ and $P \in \mathcal{P}[g] \cap \mathcal{E}[g]$ satisfy $Z^{\prime} \subseteq$ $Z \cap P$ and $g\left(Z^{\prime}\right) \geq g(Z)$. As $K$ is a partial transversal of $\mathcal{P}[g]$, we have $\left|Z^{\prime} \cap K\right| \leq|P \cap K| \leq 1$. With (5.3) for $Z^{\prime}$, this implies $|Z \backslash K| \geq\left|Z^{\prime} \backslash K\right| \geq\left|Z^{\prime}\right|-1 \geq g\left(Z^{\prime}\right)-1 \geq g(Z)-1=\hat{g}_{K}(Z)$.

Let us consider the effective set family $\mathcal{E}\left[g_{K}\right] \subseteq \mathcal{F}_{K}$ and the bunch partition $\mathcal{P}\left[g_{K}\right]$ defined for the reduction $g_{K}$. They are families on $U \backslash K$. Note that they do not necessarily coincide with $\{Z \backslash K \mid Z \in \mathcal{E}[g]\}$ and $\{P \backslash K \mid P \in \mathcal{P}[g]\}$ (see Example 3.8).

To show Proposition 3.7, we prepare the following five claims.

Claim 5.4. For every $X \in \mathcal{E}\left[g_{K}\right]$, there exists $P \in \mathcal{P}[g] \cap \mathcal{E}[g]$ such that $X \subseteq P \backslash K$.

Proof. By (5.4), there is $Z \in \mathcal{F}$ satisfying $g_{K}(X)=\hat{g}_{K}(Z)$ and $Z \backslash K=X$. Suppose to the contrary, that $X \nsubseteq P \backslash K$ for every $P \in \mathcal{P}[g] \cap \mathcal{E}[g]$. Since $Z \backslash K=X$, this implies $Z \nsubseteq P$ for every $P \in \mathcal{P}[g] \cap \mathcal{E}[g]$. Then, we have $Z \notin \mathcal{E}[g]$ by the definition of $\mathcal{P}[g]$. Since $X \in \mathcal{E}\left[g_{K}\right]$ implies $2 \leq g_{K}(X)=\hat{g}_{K}(Z) \leq g(Z)$, Claim 3.3 implies that there exist $Z^{\prime} \in \mathcal{E}[g]$ and $P^{\prime} \in \mathcal{P}[g] \cap \mathcal{E}[g]$ with $Z^{\prime} \subseteq Z \cap P^{\prime}$ and $g\left(Z^{\prime}\right) \geq g(Z)$. Then, we have

$$
Z^{\prime} \backslash K \subseteq Z \backslash K=X, \quad Z^{\prime} \backslash K \subseteq P^{\prime} \backslash K, \quad X \nsubseteq P^{\prime} \backslash K
$$

This implies $Z^{\prime} \backslash K \subsetneq X$, and hence $g_{K}\left(Z^{\prime} \backslash K\right)<g_{K}(X)$ follows from $X \in \mathcal{E}\left[g_{K}\right]$. However, (5.4), $g\left(Z^{\prime}\right) \geq g(Z)$ and $(5.5)$ imply $g_{K}\left(Z^{\prime} \backslash K\right) \geq \hat{g}_{K}\left(Z^{\prime}\right) \geq \hat{g}_{K}(Z)=g_{K}(X)$, a contradiction.

By Claim 5.4, we can observe the following structural property of $\mathcal{P}\left[g_{K}\right]$.

Claim 5.5. $\mathcal{P}\left[g_{K}\right]$ is a refinement of the partition $\{P \backslash K \mid P \in \mathcal{P}[g]\}$ of $U \backslash K$.

Claim 5.6. For $Z \in \mathcal{F}$ and $P \in \mathcal{P}[g] \cap \mathcal{E}[g]$ with $Z \backslash K \subseteq P \backslash K$, we have $\hat{g}_{K}(Z) \leq \hat{g}_{K}(P)$. In particular, if $Z \backslash K \subsetneq P \backslash K$ and $P \cap K=\emptyset$, then $\hat{g}_{K}(Z)<\hat{g}_{K}(P)$.

Proof. Take $Z \in \mathcal{F}$ and $P \in \mathcal{P}[g] \cap \mathcal{E}[g]$ with $Z \backslash K \subseteq P \backslash K$. Note that $P \in \mathcal{E}[g]$ implies $g(P) \geq 2$ by (5.1), and hence $\hat{g}_{K}(P) \geq 1$. Then, it suffices to consider the case $\hat{g}_{K}(Z) \geq 2$. Therefore, we assume $g(Z) \geq 2$. 
As $Z \in \mathcal{F}$, Claims 3.2 and 3.3 imply that there are $Z^{\prime} \in \mathcal{E}[g]$ and $P^{\prime} \in \mathcal{P}[g] \cap \mathcal{E}[g]$ satisfying $Z^{\prime} \subseteq Z \cap P^{\prime}$ and $g\left(Z^{\prime}\right) \geq g(Z)$. (In particular, $Z^{\prime}=Z$ if $Z \in \mathcal{E}[g]$.) As $K$ is a partial transversal, $\left|Z^{\prime} \cap K\right| \leq\left|P^{\prime} \cap K\right| \leq 1$. Also, $Z^{\prime} \in \mathcal{E}[g]$ implies $\left|Z^{\prime}\right| \geq 2$ by Claim 5.2. Thus, we have

$$
\emptyset \neq Z^{\prime} \backslash K \subseteq Z \backslash K \subseteq P \backslash K, \quad Z^{\prime} \subseteq P^{\prime} .
$$

As $\mathcal{P}[g]$ is a partition, these two imply $P=P^{\prime}$. Thus, $Z^{\prime} \subseteq P$, and hence 5.2 for $P \in \mathcal{E}[g]$ implies either $Z^{\prime}=P$ or $g\left(Z^{\prime}\right)<g(P)$. In the case $Z^{\prime}=P$, we have $P=Z^{\prime} \subseteq Z$ and $g(P)=g\left(Z^{\prime}\right) \geq g(Z)$, which imply $\hat{g}_{K}(Z) \leq \hat{g}_{K}(P)$ by $(5.5)$. In the case $g\left(Z^{\prime}\right)<g(P)$, we have $\hat{g}_{K}(Z) \leq \hat{g}_{K}(P)$ by $(5.6)$. Thus, the first statement is shown.

For the second statement, assume $Z \backslash K \subsetneq P \backslash K$. By $Z^{\prime} \subseteq Z$, this implies $Z^{\prime} \backslash K \subsetneq P \backslash K$, and hence $Z^{\prime} \neq P$. By the above argument, then $g\left(Z^{\prime}\right)<g(P)$. Therefore, when $P \cap K=\emptyset$, we have $\hat{g}_{K}(Z) \leq g(Z) \leq g\left(Z^{\prime}\right)<g(P)=\hat{g}_{K}(P)$. Thus, $\hat{g}_{K}(Z)<\hat{g}_{K}(P)$ is obtained.

Claim 5.7. For every $P \in \mathcal{P}[g] \cap \mathcal{E}[g]$ with $P \cap K=\emptyset$, we have $P \in \mathcal{P}\left[g_{K}\right] \cap \mathcal{E}\left[g_{K}\right]$ and $g_{K}(P)=g(P)$.

Proof. Since $P \cap K=\emptyset$, we have $P \backslash K=P$ and $\hat{g}_{K}(P)=g(P)$. By (5.4), the value $g_{K}(P)$ is defined as $g_{K}(P)=\max \left\{\hat{g}_{K}(Z) \mid Z \in \mathcal{F}, Z \backslash K=P\right\}$. Here, the maximum of the righthand side is attained by $P$ itself because any $Z \in \mathcal{F}$ with $Z \backslash K=P=P \backslash K$ satisfies $\hat{g}_{K}(Z) \leq \hat{g}_{K}(P)$ by the first statement of Claim 5.6. Thus, $g_{K}(P)=\hat{g}_{K}(P)=g(P)$. Also, the second statement of Claim 5.6 implies that, for any $X \in \mathcal{F}_{K}$ with $X \subsetneq P \backslash K=P$, we have $g_{K}(X)=\max \left\{\hat{g}_{K}(Z) \mid Z \in \mathcal{F}, Z \backslash K=X\right\}<\hat{g}_{K}(P)=g_{K}(P)$. Hence, $P$ belongs to $\mathcal{E}\left[g_{K}\right]$. Also, by Claim 5.5 and $P \backslash K=P \in \mathcal{P}[g], P$ is maximal in $\mathcal{E}\left[g_{K}\right]$. Thus, $P \in \mathcal{P}\left[g_{K}\right] \cap \mathcal{E}\left[g_{K}\right]$.

Claim 5.8. For every $P \in \mathcal{P}[g] \cap \mathcal{E}[g]$ with $P \cap K \neq \emptyset$ and $X \in \mathcal{F}_{K}$ with $X \subseteq P \backslash K$, we have $g_{K}(X)<g(P)$.

Proof. Since $P \cap K \neq \emptyset$, we have $\hat{g}_{K}(P)=g(P)-1<g(P)$. By (5.4), we have $g_{K}(X)=$ $\max \left\{\hat{g}_{K}(Z) \mid Z \in \mathcal{F}, Z \backslash K=X\right\}$, which is at most $\hat{g}_{K}(P)$ because $Z \backslash K=X \subseteq P \backslash K$ implies $\hat{g}_{K}(Z) \leq \hat{g}_{K}(P)$ by the first statement of Claim 5.6. Therefore, $g_{K}(X) \leq \hat{g}_{K}(P)<g(P)$.

We are now ready to show Proposition 3.7 .

Proof of Proposition 3.7. Take any $u \in U \backslash K$. If $u \in U \backslash \bigcup \mathcal{E}[g]$, then $u \in U \backslash \cup \mathcal{E}\left[g_{K}\right]$ by Claim 5.4. Then, $P\left[g_{K}\right](u)=P[g](u)=\{u\}$, and hence $P\left[g_{K}\right](u) \cap K=\{u\} \cap K=\emptyset$ because $u \in U \backslash K$. We also have $d\left[g_{K}\right](u)=d[g](u)=1$. Thus, the claim is proved for this case. Therefore, we assume $P[g](u) \in \mathcal{E}[g]$.

In the case $P[g](u) \cap K=\emptyset$, Claim 5.7 implies $P\left[g_{K}\right](u)=P[g](u)$ and $g_{K}(P[g](u))=$ $g(P[g](u))$. By Proposition 3.5, then we have $d\left[g_{K}\right](u)=g_{K}\left(P\left[g_{K}\right](u)\right)=g(P[g](u))=d[g](u)$. Thus, $d\left[g_{K}\right](u)=d[g](u)$ is obtained.

In the case $P[g](u) \cap K \neq \emptyset$, we have either $P\left[g_{K}\right](u)=\{u\}$ or $P\left[g_{K}\right](u) \in \mathcal{E}\left[g_{K}\right]$. If $P\left[g_{K}\right](u)=\{u\}$, then $d\left[g_{K}\right](u)=1<2 \leq g(P[g](u))=d[g](u)$ follows from Proposition 3.5 and $P[g](u) \in \mathcal{E}[g]$. Thus, $d\left[g_{K}\right](u)<d[g](u)$. If $P\left[g_{K}\right](u) \in \mathcal{E}\left[g_{K}\right]$, then $u \in P[g](u) \cap P\left[g_{K}\right](u)$ and Claim 5.5 imply $P\left[g_{K}\right](u) \subseteq P[g](u) \backslash K$. Then, $g_{K}\left(P\left[g_{K}\right](u)\right)<g(P[g](u))$ by Claim 5.8 . By Proposition 3.5, this means $d\left[g_{K}\right](u)<d[g](u)$.

Remark 5.9. The results of this paper can be extended to the setting of skew-supermodular coloring 4. To obtain the key lemma (Lemma 2.2), we used intersecting supermodularity directly only in the proof of Claim 5.1. We can observe that this claim is also true for skewsupermodular functions. Moreover, it is shown in Iwata and Yokoi [6] that Proposition 2.1 and Claim 3.1 are true for skew-supermodular functions. Therefore, Theorem 1.4 can extends to skew-supermodular functions. 


\section{Acknowledgments}

I would like to thank András Frank for his valuable comments and questions, which motivated me to work on this subject. I gratefully acknowledge Tamás Király, Kristóf Bérczi, and Satoru Iwata for their useful comments. This work was supported by JST CREST, Grant Number JPMJCR14D2, Japan.

\section{References}

[1] M. Bárász, J. Becker, and A. Frank: An algorithm for source location in directed graphs, Operations Research Letters, 33 (2005), pp. 221-230.

[2] O. V. Borodin, A. V. Kostochka, and D. R. Woodall: List edge and list total colourings of multigraphs, Journal of combinatorial theory, Series B, 71 (1997), pp. 184-204.

[3] A. Frank: Connections in Combinatorial Optimization, Oxford Lecture Series in Mathematics and its Applications, 38, Oxford University Press, Oxford, 2011.

[4] A. Frank and T. Király: A survey on covering supermodular functions, Research Trends in Combinatorial Optimization (W. J. Cook, L. Lovász, and J. Vygen, eds.), Springer-Verlag,, 2009, pp. 87-126.

[5] F. Galvin: The list chromatic index of a bipartite multigraph, Journal of Combinatorial Theory, Series B, 63 (1995), pp. 153-158.

[6] S. Iwata and Y. Yokoi: List supermodular coloring, Combinatorica, to appear.

[7] D. Kőnig: Graphok és alkalmazásuk a determinánsok és a halmazok elméletére (Hungarian; Graphs and their application to the theory of determinants and sets), Mathematikai és Természettudományi Értesitö, 34 (1916), pp. 104-119.

[8] A. Schrijver: Supermodular colourings, Matroid Theory (L. Lovász and A. Recski, eds.), North-Holland, Amsterdam, 1985, pp. 327-343. 